\title{
Getting warmer with liver transplants
}

Liver transplants have been a lifesaving therapy for decades. But innovation toward improving organ preservation and transplantation has remained remarkably limited over the years. In a welcome development for the field, a randomized trial recently reported in Nature compared the standard method of static cold storage to normothermic perfusion in liver transplantation ${ }^{1}$. The results of the study suggest that maintaining the liver by metabolically active perfusion at body temperature may be advantageous.

The list of patients waiting for a liver transplant has increased substantially in recent years. At least partly to blame is the success of liver transplantations. "Because they have been so successful, the indications are expanding to tumors: hepatocellular carcinoma and cholangiocarcinoma, even colorectal metastasis. A lot of people want to benefit from liver transplantation," says Philipp Dutkowski, head of abdominal organ transplantation at the University of Zurich. With an increase in organ demand, the motivation to use suboptimal organs, such as those from donors with cardiovascular death or other comorbidities, is gaining traction. But methods that enable preservation of these suboptimal organs have not kept pace with demand (currently, about $20 \%$ of patients on the list die waiting for a liver).

One preservation method that has received attention in recent years is normothermic machine perfusion (NMP). In contrast to static cold storage, where the organ is flushed, cooled and placed in an ice-box to reduce its metabolic activity, NMP involves perfusing the liver with oxygenated blood at normal body temperature. Animal models and a phase 1 trial have suggested that normothermic preservation may replenish ATP levels, contributing to an improvement in post-transplant survival. The approach may also allow interventions before transplant, such as defattening or treating with drugs or regenerative cell therapies, and organ function assessment.

The randomized trial by Nasralla et al. ${ }^{1}$ for the Consortium for Organ Preservation

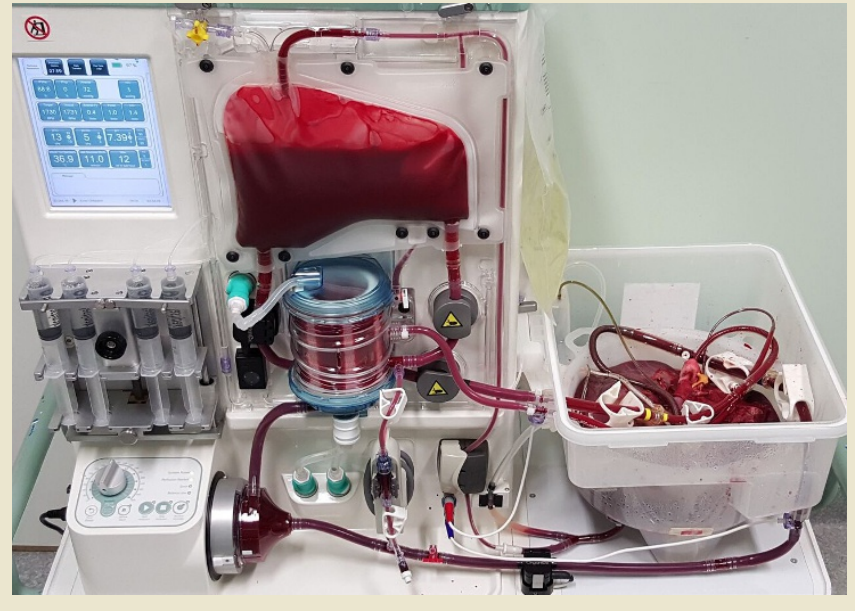

in Europe included 220 liver transplants. Although half of the organs were assigned to each preservation method, more organs stored in the cold were discarded before transplantation as compared with NMP (24.1\% versus $11.7 \%)$. The primary endpoint of the trial, peak aspartate aminotransferase (AST, a measure of liver damage) levels 7 days after transplant, was $50 \%$ lower in the NMP group as compared to the static cold storage group. Bilirubin levels were also lower in this group, providing another measure of improved post-operative graft function.

As a first of its kind, the study involved a population of patients and organs that would be expected to do well in either condition. Patients in both groups had highly successful outcomes, with no differences observed in the rate of biliary strictures, length of hospital stay (15 days) and a 95\% one-year graft survival ${ }^{1}$. "There is clear improvement [with NMP] but both sides did quite well," says Markus Selzner, surgical director of the liver transplant program at Toronto General Hospital. "The study shows that this technology is safe and probably better than cold storage, and it offers new opportunities for interventions. Next steps will need to focus on severely injured grafts."

Dutkowski is more circumspect. "At the moment, I don't see a big difference between the two arms of the study," he says. "Using AST
7 days after transplantation is not a clinically relevant endpoint." He also points out that the reasons for the lower organ discard rate in NMP are not presented in the paper ${ }^{1}$. "To show that the difference in dropout rate is meaningful, we would need to know why organs were discarded on both arms of the trial," he says. A decrease in biliary complications and graft loss within the first year would be much more impressive measures, he suggests.

The current NMP machines are heavy and bulky, and cannot be loaded on a plane, says Selzner. But this is bound to be corrected by the markets if the approach is shown to enable substantial improvements in graft survival with injured livers. More accurate measures of organ function, preserved both in the cold and warm settings, will be key to designing trials in the future and improving graft survival. In the meantime, the trial from Nasralla et al. ${ }^{1}$, in a field that has remained stagnant for decades in terms of testing and incorporating new technologies, opens up much needed discussion of avenues that will ultimately get patients off the organ wait list.

Irene Jarchum, Senior Editor

1. Nasralla, D. et al. Nature $557,50-56$ (2018). 